\title{
A Case for Cognitive Models in Visualization Research Position paper
}

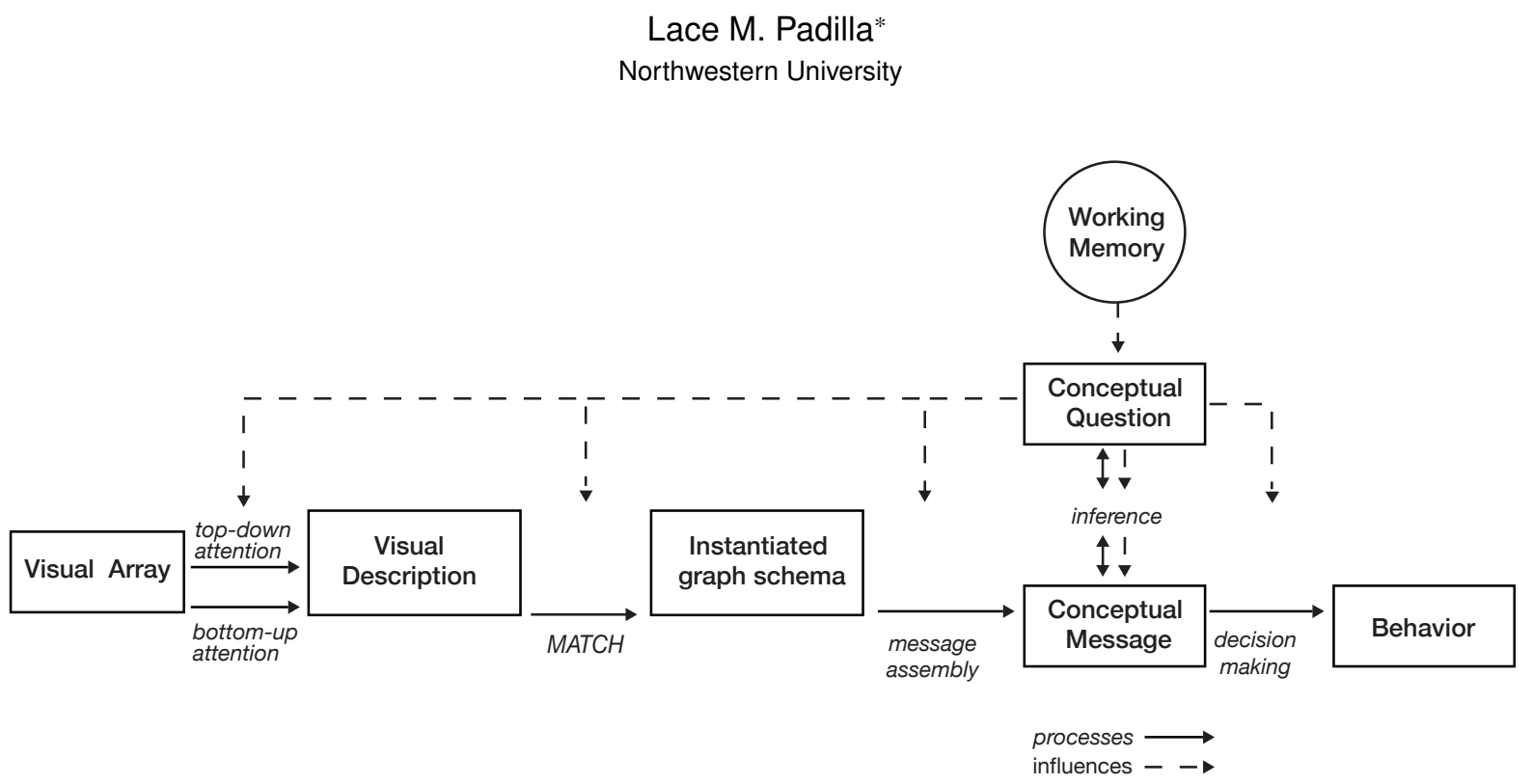

Figure 1: Path model illustrating the cognitive process of making decision with visualizations [41]. Reprinted with permission from "Decision-making with Visualizations: A Cognitive Framework Across Disciplines." by Padilla, L., Creem-Regehr, S. H., Hegarty, M., Stefanucci, J. K. (2018). Cognitive Research: Principles and Implications, 3(1), 29.

\section{Abstract}

The visualization community has seen a rise in the adoption of user studies. Empirical user studies systematically test the assumptions that we make about how visualizations can help or hinder viewers' performance of tasks. Although the increase in user studies is encouraging, it is vital that research on human reasoning with visualizations be grounded in an understanding of how the mind functions. Previously, there were no sufficient models that illustrate the process of decision-making with visualizations. However, Padilla et al. [41] recently proposed an integrative model for decision-making with visualizations, which expands on modern theories of visualization cognition and decision-making. In this paper, we provide insights into how cognitive models can accelerate innovation, improve validity, and facilitate replication efforts, which have yet to be thoroughly discussed in the visualization community. To do this, we offer a compact overview of the cognitive science of decision-making with visualizations for the visualization community, using the Padilla et al. [41] cognitive model as a guiding framework. By detailing examples of visualization research that illustrate each component of the model, this paper offers novel insights into how visualization researchers can utilize a cognitive framework to guide their user studies. We provide practical examples of each component of the model from empirical studies of visualizations, along with visualization implications of each cognitive process, which have not been directly addressed in prior work. Finally, this work offers a case study in utilizing an understanding of human cognition to generate

*Department of Psychology, Evanston, IL, USA. e-mail: Lace.M.K.Padilla@gmail.com a novel solution to a visualization reasoning bias in the context of hurricane forecast track visualizations.

Index Terms: Cognitive Model-Cognition-Decision MakingHuman Centered Design—Replication-Visualization DesignEvaluation Methods-Innovation-Validity

\section{INTRODUCTION}

At their best, visualizations of data effortlessly reveal the true nature of information. Essential patterns in data may go unnoticed until we use visualizations to understand data in new ways [6]. While understanding how the mind reasons with visual information is key to accurately predicting how a viewer will make decisions with a visualization, there has been a general lack of focus on cognition in visualization science $[26,33,41]$. Visualization research that fails to incorporate human reasoning may stifle innovation, reduce validity, and hinder replication efforts.

The lack of incorporation of a modern understanding of mental processing is likely due to the absence of a clear illustration of how we make decisions with visualizations, aimed at informing the visualization community. This paper provides a high-level overview of modern cognitive theories for how humans make decisions with visualizations. We use a recently published cognitive model to illustrate contemporary theories of the organization of the visualization decision-making process, and then expand on how this work specifically applies to visualization research. In this paper, we provide insights into how cognitive models can accelerate innovation, improve validity, and facilitate replication efforts, which have yet to be thoroughly discussed in the visualization community. To illustrate the application of a cognitive model for decision-making with visualizations, we offer examples from empirical studies that show how specific cognitive processes can influence how effectively people use visualizations. As a case study, we demonstrate the use of the Padilla et al. [41] cognitive model to identify the specific 
decision-making process that was responsible for viewers' misinterpretation of ensemble hurricane forecast track visualizations [45]. Identifying the potential cognitive processes responsible for reasoning errors allowed us to explore novel solutions that we would not have considered otherwise. In the end, we were able to reduce the reasoning errors by making changes to the hurricane forecast visualization technique, which could improve people's decisions when making time sensitive and high-risk decisions about impending storms $[36,52]$. The unique contribution of this work is the focused discussion of how pressing issues in visualization research (i.e., innovation, validity, and replication) can be supported with a thorough understanding of how the human mind processes visualizations. Cognitive frameworks provide a human-centered structure for approaching these problems. Beyond theoretical insights, this paper expands on our prior work by curating practical examples for the visualization community of how each component of the cognitive model relates to visualization research.

\section{Why USE A COGNITIVE MOdEL?}

Cognitive models are summaries of mental process theories. They can include a particular theory or an integration of multiple theories and approaches. Cognitive models can be illustrated as process diagrams, such as in Figure 1. Process diagrams illustrate the overarching framework of a cognitive theory and can be useful to summarize large bodies of research. For example, the process diagram in Figure 1 illustrates the mental processes associated with visualization decision-making - beginning with viewing a visualization (the visual array) and concluding with a behavior based on a decision. The boxes and paths in between the visual array and behavior denote specific cognitive mechanisms, processes, and influences that researchers have extensively examined in numerous domains. Although each component of this model denotes entire fields of research and is obviously an oversimplification, it provides readers with a broad overview of current conceptualizations of the visualization decision-making process.

Research on visualization decision-making that is not informed by underlying cognitive processes may undermine innovation by failing to examine solutions based on cognitive science. An understanding of the full space of solutions is vital for maximizing innovation. Leaving out relatively obvious options based on a lack of understanding of cognition drastically reduces the decision space and thus constrains innovation. Considering the importance and impact of decisions with visualizations, researchers need to build upon previously established theories of how the mind processes visualizations to ensure that the best solutions are identified as quickly as possible.

Cognitive models can also be useful in evaluating the validity of research. There are various forms of validity, each of which is important for the visualization community to consider. The term ecological validity refers to how closely the conditions of an experiment match real-world conditions [9]. As with any empirical research, there is a trade-off between experimental control and ecological validity, as illustrated in Figure 2. To make an optimal choice between experimental control and ecological validity, researchers need to understand the cognitive processes at work when real users are performing the task in a natural setting. If the experimenters know which cognitive conditions are fundamental to the task, then they can determine which elements could be changed to increase experimental control. The second type of validity is external validity, which refers to the ability to generalize contexts and groups other than the ones tested in the original study $[3,5,51]$. For example, a study might have groundbreaking results that apply to only a small group of people. On the other hand, a study might have a small impact but apply to the majority of people and contexts. Cognitive models such as that of Padilla et al. [41] often illustrate how individual differences in knowledge or expertise influence cognition. If researchers
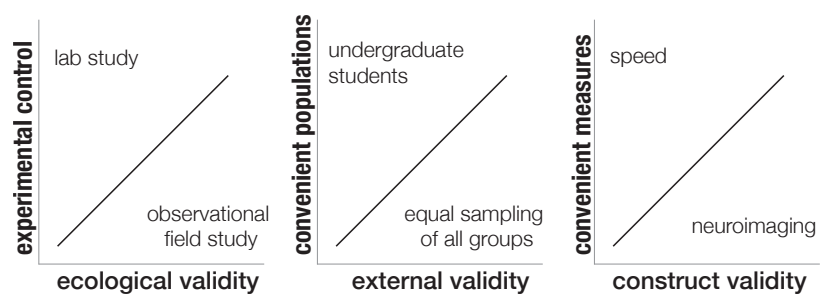

Figure 2: Example of specific types of validity: ecological, external, and construct. This figure illustrates the trade-off between validity and other components of an experiment.

believe that knowledge-driven processing plays an important role in their study, then they should either provide participants with training, in an attempt to equalize viewers' knowledge, or focus on a group of experts, which could reduce external validity. The final type of validity is construct validity, which refers to the ability of a study to measure what it claims to measure [14]. For example, some visualization studies use a task-based approach and measure speed and accuracy then claim that these measures are proxies for mental effort [66-69]. In contrast, a cognitive approach offers more direct measures of mental effort that have yet to be widely adopted by visualization studies, such as neuroimaging, pupillometry, or dual-task paradigms [7]. Visualization researchers who are interested in making claims about mental effort should consider adopting more direct measures of working memory. For example, other fields have demonstrated that working memory can be effectively measured during tasks such as virtual navigation and spacial abilities measures [21]. Working memory measures provide greater construct validity than measures of speed and accuracy. By using a cognitive model, researchers can identify which cognitive component they are interested in and then utilize the most direct measures of testing it. Using a cognitive model, at a minimum, helps researchers make more informed decisions about the trade-offs between experimental control vs. ecological validity, populations of convenience (such as undergraduate students at elite universities) vs. external validity, and measures of convenience (such as speed and accuracy) vs. construct validity (as seen in Figure 2).

An additional benefit of utilizing a cognitive model is the ability to conduct conceptual replications more effectively. Conceptual replication is when researchers create a study intended to reveal the same phenomenon, results, or cognitive processes of previous research [61]. In the context of visualization user studies, researchers must have a clear understanding of the underlying mental processes that produce the specific finding to attempt to replicate the result conceptually. One way to conduct a conceptual replication is to utilize a cognitive model to identify the specific components or interaction of components in the visualization decision-making process that produced the original finding. If a researcher can identify the responsible components or interactions, then it becomes more clear which components of an experiment can be changed and which need to remain constant to enact a conceptual replication. Note that a full discussion of the types of replication is beyond the scope of this paper, but researchers are engaged in an ongoing debate about the merits of various types of replication techniques [10,46,57,61]. One view argues that direct or exact replication is the gold standard for evaluating the size and reliability of a finding [46, 57]. Exact replication is when a group of researchers attempts to duplicate a previous study, using identical methods, to determine if the secondary study reveals the same findings. Researchers must use the same population, experiment design, data collection procedures, stimuli, and data analysis procedures as the original study to ensure that any differences between the results are not due to changes in the experiment $[46,57]$. However, the nature of visualization re- 
search often makes direct replication unattainable. For example, if a study utilizes expert participants, it might be impossible to find a comparable group of experts to conduct a direct replication as the specific training or organizational norms might vary between groups of experts. In some cases, a conceptual replication would be the viable option, and in these cases, a solid understanding of the mental processes associated with an experiment is required [61].

In sum, cognitive models provide a high-level overview of mental processing and can be used to bolster innovation, improve validity, and facilitate replication efforts. The following sections offer a description of one cognitive model that visualization practitioners can use as an organizing framework for the vast body of cognitive science on visualization decision-making.

\section{Cognitive Model}

The model proposed by Padilla, Creem-Regehr, Hegarty, and Stefanucci [41] is an integrative model that synthesizes theories of visualization comprehension $[26,34,49,55,68]$ and decision-making $[13,17,23,59,63]$. The following section will detail each mechanism (square), process (solid path), and influence (dashed path) of the Padilla et al. [41] model (see Figure 1) for a visualization audience. Each section includes a specific description of visualization applications of the cognitive mechanism. As the aim here is to provide a high-level overview of the visualization decision-making process, each section is a considerable oversimplification. However, for those interested in diving deeper into a specific topic, we offer recommended reading on each cognitive mechanism.

\subsection{Visual Array}

The visual array is the unprocessed neuronal firing in response to visualizations [49]. The anatomy of the eye, and more broadly the visual system, profoundly influences the way we reason with visualizations. The visual system refers to the complicated relationship between the eyes and brain that facilitates vision [24]. By understanding the visual system, we have the building blocks for understanding how people reason with visualizations. This research is outlined by comprehensive texts, including those by William Thompson, Roland Fleming, Sarah Creem-Regehr, and Jeanine Stefanucci [62] and Tamara Munzner [39].

The anatomy of the eye can influence viewers' interpretation of visualizations in a variety of ways [62]. One relevant application is the perception of color. Color is a commonly used encoding channel [39], and the organization of color can change a viewers' perception of a specific color, known as simultaneous contrast [24]. Simultaneous contrast is when the surrounding colors influence our perception of a specific color, which arises from the relationship between neighboring neurons in the visual system [4,24,62]. For a simple example see Figure 3, which demonstrates the Mach Band illusion where edges of the rectangles appear to have dark and light coloration even though each rectangle is a single gray value [50]. This illusion is created by the organization of neurons in the eye, which is termed lateral inhibition [24].

The Mach Band illusion and other similar perceptual phenomena can have significant effects on how viewers reason with visualizations. For example, Figure 4 shows sections of a digital elevation map (DEM) used as stimuli in Padilla et al. [42], with A showing a continuous grayscale encoding and $\mathrm{B}$ showing the same data but binned at 40-meter intervals. In this study participants completed 12 tasks ranging in complexity from look-up to feature integration and comparison tasks, while viewing either a continuously encoded DEM or DEMs with various binning intervals (10-, 20-, 30-, 40meter intervals). The researchers were interested in evaluating how binning intervals influenced performance on a wide range of geospatial tasks. The overall takeaway from this study was that there were no cases where the continuous encoding facilitated better accuracy than one of the binning techniques [42]. One possible reason for the

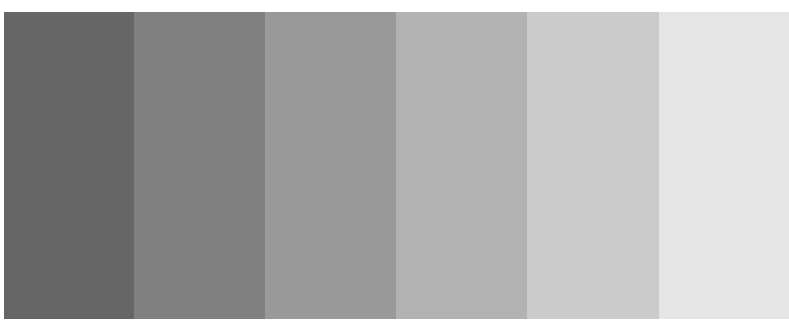

Figure 3: Example Mock Band illusion, where the edges of the gray bands create the perceptual effect of lightness on the relatively lighter gray edge and darker on the relatively darker gray edge, even though each section is a discrete gray [38].

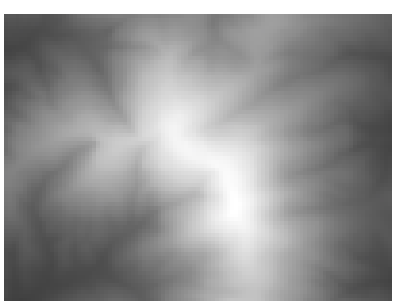

(a)

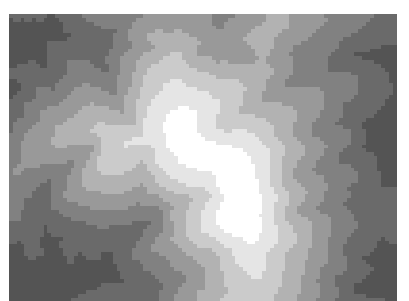

(b)
Figure 4: $\mathrm{A}$ is a continuous grayscale encoding of a portion of a digital elevation map, which was used as stimuli in Padilla et al. [42]. B is the same data but binned at 40-meter intervals. Reprinted with permission from "Evaluating the impact of binning 2D scalar fields" by Padilla, L., Quinan, P. S., Meyer, M., Creem-Regehr, S. H. (2016).IEEE transactions on visualization and computer graphics, 23(1), 431-440.

superiority of the binning methods could be the Mach Band effect created by the bins. Notice in Figure $4 \mathrm{~B}$ that a Mach Banding effect dramatizes the edges of the bins. Mach Banding effects might also increase the salience of borders of choropleth maps with sequential color encodings.

\subsection{Bottom-up Attention}

The amount of information in the world is greater than our visual systems' limited ability to perceive it. Attention is the mechanism our visual systems use to select the information to perceive [65], which is a topic that researchers have studied extensively in neuroscience, physiology, psychology, and computer vision [29]. Visualizations can draw our attention to specific salient features. Salience is the degree which a visual feature will stand out from its surrounding elements [29]. A variety of approaches exist for computing the salience of an image, which commonly identifies local changes in color, intensity, orientation, motion cues, structures, and gradients $[8,11,29]$. This type of stimulus-driven attention is bottom-up and is thought to be pre-conscious or involuntary $[12,53]$.

Numerous studies have demonstrated that when incorporated into visualization design, salience can guide viewers' bottom-up attention to task-relevant information, thereby improving performance $[18,19$, $27,45,54,60,72]$. To evaluate which elements of a visualization are more salient, consider utilizing the Itti et al. [28] model, which has been evaluated with eye tracking data.

One way to utilize bottom-up attention when developing visualizations is to capitalize on the pop-out effect and perceptual grouping. Munzner [39] describes how many visual channels support the fast identification of mismatched visual features (pop-out) and the visual clustering of features (Gestalt laws of perceptual grouping), including color, shape, proximity, and tilt, among many others (see Figure 5 for examples). The broad goal of utilizing pop-out and Gestalt laws is to create a visual hierarchy of information, and in doing 


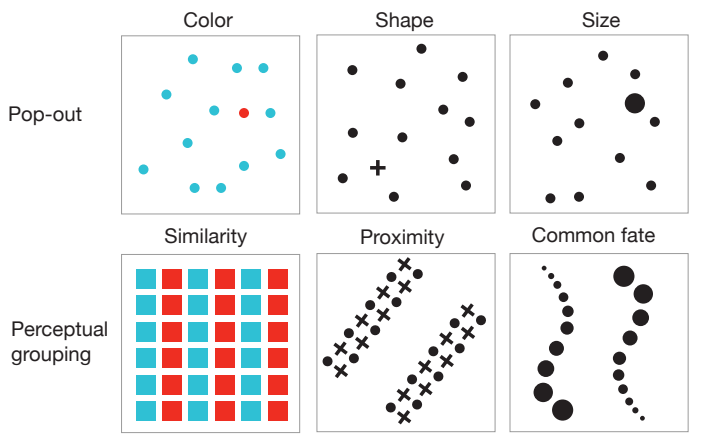

Figure 5: Examples of pop-out effects and perceptual grouping.

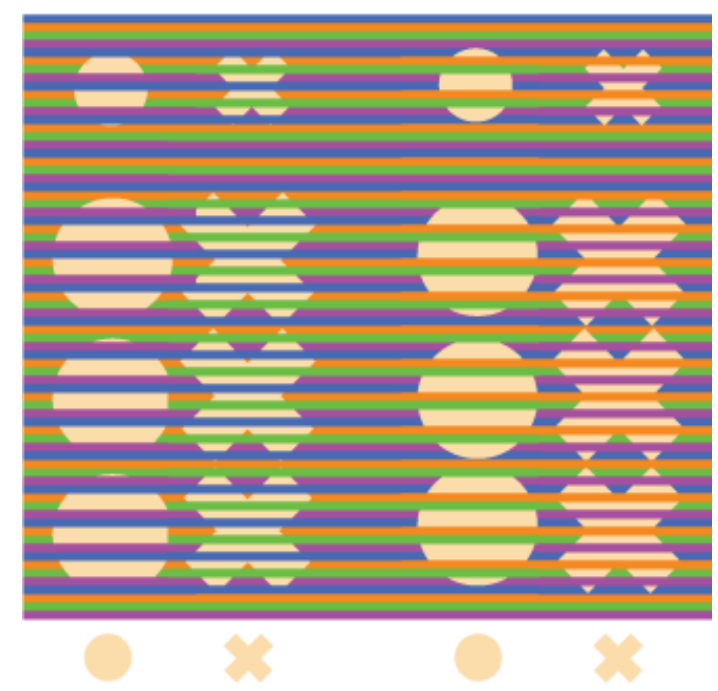

Figure 6: An optical illusion in which the colors of the circles and Xs are the same but appear to be different, based on a Munker-like illusion developed by David Novick and Gestalt grouping principles. The shapes below the illusion are all the same color and are the same color as the shapes within the illusion.

so, direct viewers' bottom-up attention to task-relevant information. Researchers have employed low-level vision research to develop colormaps that enhance the pop-out effects [70] and utilize perceptual grouping to help viewers quickly identify similarities between text and imagery [2]. An interesting example of the strong effects of perceptual groupings is found in Figure 6. How do you see the shapes grouped in this image? By color, shape, proximity, or size? You likely see some combination of each with color playing a primary role. Interestingly, the color of the circles and Xs are the same as illustrated by the beige-colored objects below the illusion. Through a combination of a Munker-like illusion developed by David Novick and Gestalt grouping principles, the illusion creates the perception that the shapes are different colors.

Note that the terms bottom-up and top-down are commonly used to refer to processes that are automatic, unconscious, and physiologically based (bottom-up) and those that incorporate knowledge and conscious processing (top-down). The Padilla et al. [41] model is in line with previous theories in asserting that visualization decisionmaking is an integration of both bottom-up and top-down processing $[26,34,49,55,68]$.

\subsection{Top-down Attention}

Top-down attention is when viewers deliberately direct their gaze to specific features of a visualization based on knowledge about their task [53]. Our ability to direct our attention is limited, and researchers find that these limitations significantly influence our ability to reason with visualizations [25]. For example, a viewer might direct her attention to the legend in a visualization because she knows that the legend contains valuable information. In contrast, someone without a formal education might not have learned the function of a legend and not direct her attention to it. When we consciously direct our attention, effort is required. We will discuss working memory at length in Section 3.4, but broadly, working memory is the control mechanism that allocates mental effort [13]. For further reading on the long history and evolution of bottom-up and top-down attention theory see The attention system of the human brain: 20 years after, by Steven Petersen and Michael Posner [48].

Fabrikant et al., [18] and Hegarty et al. [27] demonstrated the relationship between bottom-up and top-down processing in a study with temperature and pressure meteorological forecast maps. Hegarty et al. found that when they manipulated the salience of the temperature encoding (color) or the pressure encoding (isolines), the more salient features drew viewers' attention (as measured by eye fixations) but did not influence their performance. The researchers then trained participants on how to effectively interpret the features in the weather map and found that more participants directed their attention to the task-relevant features and their judgments improved [18,27]. This example shows how using using saliency to create a hierarchy can help in directing viewers' bottom-up attention, but it may not be enough to improve their performance. Viewers may also need to learn the task-relevant features of the visualization in order to also direct their top-down attention (see also, [18]).

\subsection{Working Memory and Conceptual Question}

The conceptual question is the task or question the viewer hopes to answer by interpreting the visualization [49]. To complete the task, viewers commonly need to apply deliberate mental effort, which requires working memory. The definition of working memory is the subject of an ongoing debate. We shall adopt the definition used by Cowan [13], that working memory consists of a multicomponent system that maintains a finite amount of information for a short period and controls the use of stored information. A central executive controls the multicomponent functions of working memory and can, among other functions, control top-down attention [1,13,47]. Working memory also holds information in a visuo-spatial temporary store, which is where information can be held for a short period without being stored in long-term memory [1]. In order to focus on task-relevant information rather than on distracting information, working memory is used to control attention and suppress automatic responses $[32,56]$. An essential characteristic of working memory for visualization researchers to note is that working memory is capacity limited, which means that working memory is a finite resource that can be overly taxed by unnecessary processing or by suppressing automatic responses.

As indicated by the dashed lines in Figure 1, working memory can be used to aid in the majority of visualization decision-making processes. The Padilla et al. [41] model illustrates how, with deliberate application of working memory, we can make slower, effortful, and more strategic decisions with visualizations. On the other hand, if we do not apply working memory, we can also make fast and computationally light decisions. The theory that proposes a distinction between fast and slow processing was developed by researchers in decision science and behavioral economics (for a review of the dual process theory of decision-making, see [17]; and for a review of dual process theory in the context of visualizations, see [41]).

Many visualization studies assert that one of the critical reasons to visualize information is to reduce mental effort $[6,26]$. By allow- 
ing our visual system to identify complex relationships in visually presented information, we free up working memory-also known as augmenting decision-making [26]. Using this perspective, studies commonly claim that a visualization technique may reduce mental effort $[15,22,64,66-69,73]$. These studies use accuracy and task completion time to infer working memory demand. However, direct measures of working memory exist, such as pupillometry and behavioral manipulations (see Padilla et al. [41] for an overview of how to measure working memory demands in a visualization task). We propose that visualization designers interested in evaluating the relative working memory demand of their visualization should directly measure working memory. Indirect measures of working memory, such as accuracy, may not be sensitive enough to detect significant changes in working memory that could have large-scale impacts on task performance outside the lab.

\subsection{Visual Description}

The visual description is the mental encoding of the visual stimulus [49]. Interestingly, the visual description is not identical to the visual array. The visual description includes only the information the viewers perceived.

Researchers have dramatically demonstrated the differences between the visual array and visual description by showing that expert radiologists commonly do not perceive a gorilla that the experimenters superimposed on chest CT images [16]. Drew, Võ, and Wolfe found that because radiologists are focused on identifying cancerous nodules, they may not perceive a cartoon gorilla that is 48 times larger than the average cancerous nodule. Many of the radiologists looked directly at the gorilla but did not perceive it, and thus the gorilla was not included in their visual description. This phenomenon, termed inattentional blindness, is observed in many contexts $[16,40,58]$.

\subsection{MATCH and Instantiated Graph Schema}

A graph schema is a graphic convention or prior knowledge about a visualization that a viewer uses to help interpret the visualization [49]. The matching process occurs when a viewer searches in longterm memory for a schema that matches a visualization. If she remembers a relevant schema, she updates the visual description with the additional information provided by the schema, thus instantiating the graph schema. For example, if a viewer sees a bar chart, she may remember the conventions for the $\mathrm{X}$ - and $\mathrm{Y}$-axis and use that information to interpret the graph. See Pinker [49] to learn more about graphic schemas.

Cognitive Fit Theory is used by researchers to consider the mental effort associated with understanding a visualization [67]. This theory suggests that viewers compare a mental schema to the visual description, and if there is not a complete match, then viewers must mentally transform the visual description. As mental transformations increase processing steps, time to complete the task, and opportunity for errors, it is desirable to reduce mental transformations. Numerous visualization studies use Cognitive Fit Theory to consider how to improve visualizations $[64,66,67,69]$.

Common visualization reasoning errors occur during the graph schema matching process when viewers accidentally use the wrong graph schema. For example, Joslyn and LeClerc [30] found that when participants viewed mean temperature forecasts with error bars, they incorrectly believed that the error bars represented highand low temperatures. The authors proposed that viewers incorrectly used the mental schema for high- and low-temperature forecasts because they looked similar. Participants continued to use the wrong schema despite a key detailing the correct way to interpret the forecasts. Other work also finds that even when given instructions on the correct mental schema to use, viewers rely on the mental schema that most closely visually matches the visualization [30,41,45]. Visualization designers should not expect viewers to use any other mental

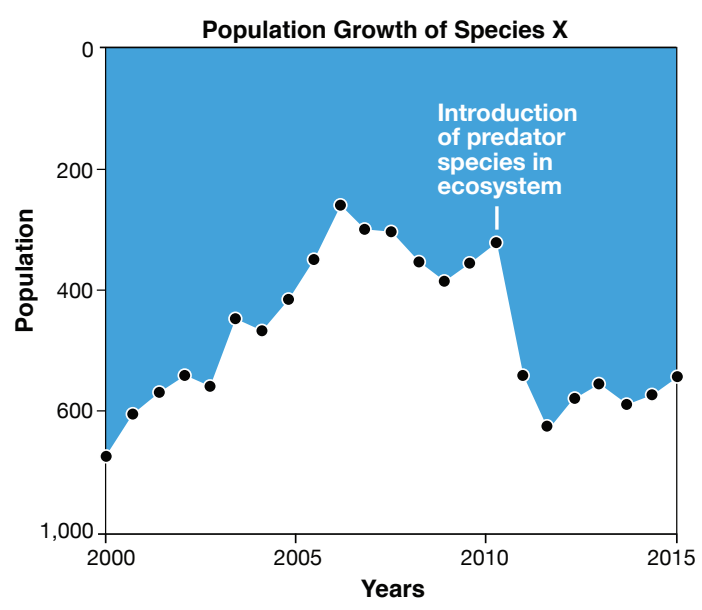

Figure 7: Reversed Y-axis figure showing the relationship between the population of species $X$ and the introduction of a predator species. Christine Chan of Reuters created the graph that inspired this one with the $\mathrm{Y}$-axis reversed ordered, which illustrated the relationship between Florida's Stand Your Ground law and firearm murders [35]. Reprinted with permission from "Decision-making with Visualizations: A Cognitive Framework Across Disciplines." by Padilla, L., Creem-Regehr, S. H., Hegarty, M., Stefanucci, J. K. (2018).Cognitive Research: Principles and Implications, 3(1), 29.

schema than the one that looks most similar to the visualization. With that said, many open questions about the specific nature of the matching process remain. Additional empirical work is needed to more specifically detail the relationship between the features in a visualization and features in a remembered graphic schema.

\subsection{Message Assembly, Inference, and the Conceptual Message}

The message assembly process is how the instantiated graph schema is integrated with the visual description to form viewers' mental representation of the visualization. The resulting mental representation is the conceptual message. In order to answer the conceptual question, the viewer may need to mentally transform or perform other mental computations on the mental representation of the visualization, which occurs in the inference process.

An example of an unnecessary mental transformation is illustrated in Figure 7 [41]. Figure 7 depicts a fictional relationship between the population growth of Species $\mathrm{X}$ and a predator species. If you look briefly at the figure, it may appear that the introduction of the predator species is correlated with a drop in the population of Species X. However, notice that the Y-axis is incorrectly reverse ordered, which violates our mental schema for graphs. Using working memory, you may be able to mentally reorder the values on the Y-axis to match the standard mental schema for graphs. However, this mental transformation is difficult, and if you do not notice the violation or do not want to work that hard, you might come to the wrong conclusion about the figure. When the viewer is forced to mentally transform the visualization, processing steps are increased, which may increase errors, time to complete a task, and demand on working memory [69]. Reversing the Y-axis may seem like a ridiculous visualization choice that no one would do in the real world. However, Figure 7 was inspired by an infamous visualization from Reuters that showed gun deaths in Florida over time and noted when the Stand Your Ground Law was enacted [35]. If a viewer briefly views the Reuters visualization, it would appear that the introduction of the Stand Your Ground law correlated with a decrease in gun deaths. However, because the Y-axis is reverse ordered, the opposite interpretation is correct. It is possible that the visualization designer 
was aware of how difficult mental transformations are and further assumed most people would not think to confirm that the visualization aligned with their mental schema for graphs. This type of intentional manipulation of mental schema assumptions is relatively rare. However, accidentally requiring the viewer to make unnecessary mental computations is common, particularly when attempting to visualize abstract information, such as uncertainty [36, 37, 44]. The issue of unintentionally requiring superfluous mental transformations highlights the importance of understanding the cognitive process of visualization decision-making.

\subsection{Decision-making and Behavior}

The final stage of the Padilla et al. [41] model includes the decisionmaking process and resulting behavior. The decision step can be completed using either working memory, which produces a slower decision, or quickly with negligible working memory [17]. Researchers propose that fast decisions commonly utilize heuristics or rules of thumb $[23,31]$. We use these mental short cuts to avoid reanalyzing every situation. Some heuristics are helpful and reduce processing demands [23]. For example, we assume that all graphs have correctly ordered Y-axes, which saves us time and energy $99.99 \%$ of the time. However, we run into issues when we apply a heuristic in the wrong context, as is the case in Figure 7. In order to override a heuristic, we must use working memory to inhibit the rule of thumb and effortfully consider the information. In a perfect world, visualizations would elicit fast and accurate judgments using helpful heuristics. However, because the average person has little experience using visualizations, as illustrated by the one-third of the US population that has surprisingly poor graph literacy [20], it is likely that the average viewer will use incorrect heuristics to interpret novel visualizations. Visualization designers should consider the trade-off between innovation and the catch-up time the general public will need to develop appropriate mental schemas to accurately interpret cutting-edge visualization techniques. For further reading on the dual process model for decision-making, see Evans [17].

Decision science has identified many common heuristics [31]. Researchers have found that visualizations can be used to help people overcome some heuristics that hinder decision-making, such as anecdotal evidence bias [19] and side effect aversion [71, 72]. Researchers find that they can reduce these biases by visually representing probabilistic data $[19,71,72]$.

\section{Case study in using a cognitive model}

To illustrate the utility of using a cognitive model to ground visualization research in cognitive theory, the following section will detail a prior experiment that evaluated ensemble hurricane track forecast visualizations. Our prior work found that an ensemble visualization technique produced fewer misinterpretations than the Cone of Uncertainty, which is currently used by the National Hurricane Association to depict storm trajectory [52]. More recently, we developed a modeling technique that outperformed our prior approaches by selectively sampling ensemble members while preserving the properties of the distribution [37], (see Figure 8). The new technique allowed for annotation of the paths to include size and intensity. Viewers were asked to rate the amount of damage that would occur to an oil rig in the area of the hurricane forecast. We found that users could effectively incorporate both the size and intensity information from the visualization into their judgments. Additionally, we found an influence of the number of paths displayed [37].

As this type of ensemble hurricane forecast visualization technique was relatively new, we wanted to test if it elicited any adverse effects on decision-making before fully endorsing it. To identify the possible adverse effects of the ensemble display, we focused on the mental schema portion of the visualization decision-making model. It occurred to us that given that this type of ensemble was new, viewers might not know the correct mental schema for interpreting the

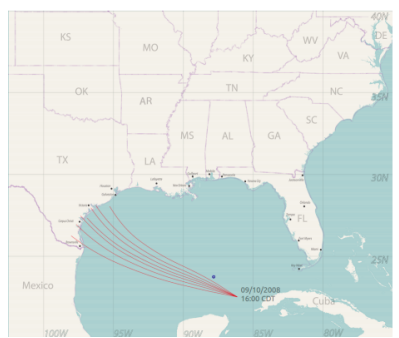

(a) 7 tracks, unannotated

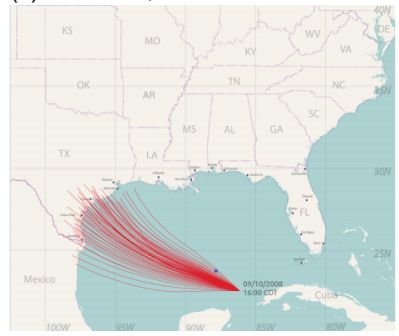

(c) 63 tracks, unannotated

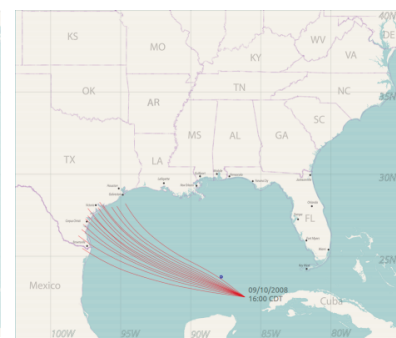

(b) 15 tracks, unannotated
Figure 8: Example of the stimuli tested in Liu et al. [36]. Reprinted with permission from "Visualizing uncertain tropical cyclone predictions using representative samples from ensembles of forecast tracks." by Liu, L., Padilla, L. Creem-Regehr, S. H., House, D. (2018), IEEE transactions on visualization and computer graphics.

ensemble display. After surveying widely available visualizations that looked similar to the ensemble display, we realized that viewers might be using a mental schema for navigation routes. We hypothesized that, as in alternative navigation routes shown in applications such as Google Maps, viewers might see each hurricane path as a deterministic route (deterministic construal error [30]). However, for the specific modeling technique that we used, each paths is a subset of the runs of the model [36]. The overall distribution of paths is intended to show the uncertainty in the forecasted path of the storm rather than specific paths that the hurricane could take. To test if viewers employed a deterministic route schema, we developed a task to test a "collocation" visual-spatial bias, which we predicted would result from the schema. The collocation effect is when viewers assign too much weight to the importance an ensemble member when it is collocated with their point of interest. For example, if a viewer believes that the hurricane paths are deterministic and is shown only three paths (one of which hits her town), she might assume the storm has a $33 \%$ chance of hitting her home. However, in the same scenario, if she is shown 20 paths and one hits her home, she might conclude that the chance the storm will impact her is only $5 \%$, which is a mental schema error. Although not tested directly, our prior work demonstrated that viewers were sensitive to the number of tracks displayed [37] and to cases where an ensemble member was collocated with the point of interest [45].

We tested the hypothesis that increasing the number of lines would reduce the over-weighting of an ensemble member that directly hit a viewer's point of interest. To do this, we created artificial hurricane forecast images that closely visually match the images used in Liu et al. [37] but that allowed for greater experimental control. In the displays we generated, either one path intersected an off shore oil rig (indicated with a black dot) or the oil rig remained in the same location, but the previously collocated path was moved to the opposite side of the distribution (see Figure 9). This experimental design allowed for a more direct evaluation of the collocation of the oil rig and hurricane track than in our prior work [45]. We tested the influence of $9,17,33$, and 65 hurricane paths on the collocation effect. To control for changes to the distribution that may occur 


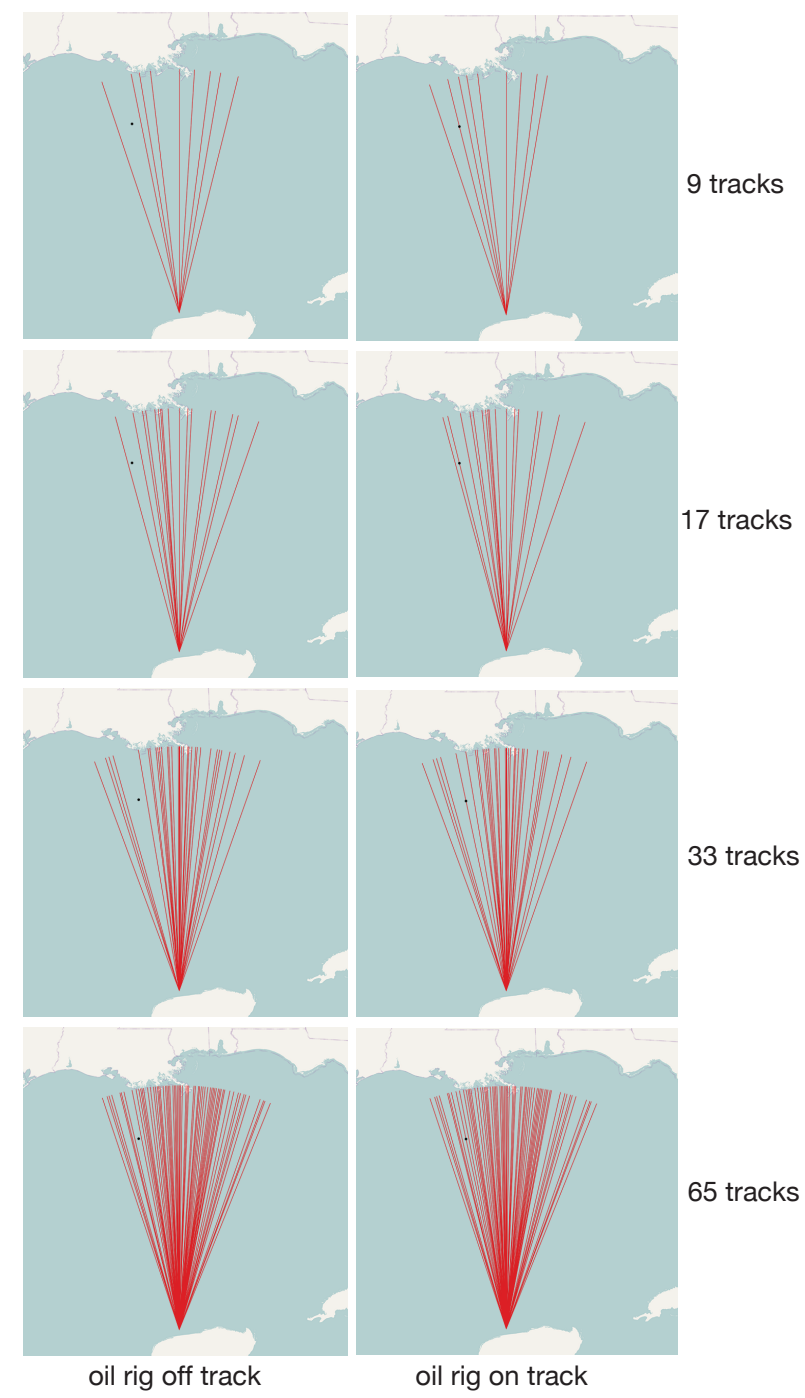

Figure 9: Example of the stimuli tested in Padilla et al. [43].

from moving one of the tracks, we generated straight paths and then reflected the paths over the center line. The reflection resulted in mirror images of each stimulus, which allowed for counterbalancing of unintended changes to the distribution. Using the same task as in Liu et al. [37], participants who were shown images with 9 hurricane paths demonstrated the most substantial bias, increasing their damage ratings by 1.7 on a Likert scale (ranging from 1-7) when an ensemble member intersected the oil rig location compared to when it did not. We were able to significantly reduce this bias by showing viewers images with 17, 33, and 65 hurricane paths [43].

In this case study, we examined the influence of a specific component of the cognitive model and in doing so discovered the source of one reasoning error with ensemble hurricane forecast visualizations. The cognitive framework gave us a roadmap for possible locations of errors and guidance for developing a task that tested a specific cognitive process. If we had not focused on evaluating mental schemas, we would not have examined the collocation effect, and we might have prematurely endorsed this visualization technique. Further, we would not have considered how the number of ensemble members represented could influence reasoning errors without careful reflection on cognitive models. Selecting the appropriate number of ensemble members is an important issue that would have been ill-considered without the incorporation of mental schemas into this work. Finally, the Padilla et al. model [41] proposed two methods for decision-making (Systems 1 and 2). Based on these insights, we elected to find a visualization solution that did not require the viewer to use additional working memory (System 1). The Padilla et al. [41] model helped us to consider leveraging the heuristics that viewers were naturally using to improve reasoning with visualizations, which is an overarching goal throughout the visualization community.

\section{CONCLUSION}

It is vital to understand how our minds process visual information when evaluating visualizations and developing new visualization techniques. Cognitive models are helpful for providing an overview of contemporary theories and can be used to ground visualization research in empirically validated findings. We detailed the visualization application of each component of the most up-to-date cognitive model for decision-making with visualizations [41]. This paper is the first to discuss how pressing topics in visualization research, including innovation, validity, and replication are supported by an understanding of how the human mind processes visualizations. Further, we have expanded on the Padilla et al. [41] model by illustrating each component with relevant examples to the visualization community. Finally, we used a case study to illustrate how to employ a cognitive model to ground and guide visualization research. Given the importance and large-scale impact of visualizations, their cognitive underpinnings should be of supreme importance to visualization researchers to ensure that visualizations are actually revealing the true nature of information.

\section{ACKNOWLEDGMENTS}

The author wishes to thank Sarah Creem-Regehr, Mary Hegarty, William Thompson, Jeanine K Stefanucci, Donald House, and Le Liu. This work was supported in part by the National Science Foundation under Grants 1212806, 1810498, and 1212577.

\section{REFERENCES}

[1] A. Baddeley. Working memory. Science, 255(5044):556-559, 1992.

[2] J. Bae and B. Watson. Reinforcing visual grouping cues to communicate complex informational structure. IEEE transactions on visualization and computer graphics, 20(12):1973-1982, 2014.

[3] L. Berkowitz and E. Donnerstein. External validity is more than skin deep: Some answers to criticisms of laboratory experiments. American psychologist, 37(3):245, 1982.

[4] C. A. Brewer. Evaluation of a model for predicting simultaneous contrast on color maps. The Professional Geographer, 49(3):280-294, 1997.

[5] B. J. Calder, L. W. Phillips, and A. M. Tybout. The concept of external validity. Journal of Consumer Research, 9(3):240-244, 1982.

[6] M. Card. Readings in information visualization: Using vision to think. Morgan Kaufmann, 1999.

[7] S. Castro, D. Strayer, D. Matzke, and A. Heathcote. Cogntiive workload measurement and modeling under divided attention. JEP:General, in press.

[8] M.-Y. Chan, Y. Wu, W.-H. Mak, W. Chen, and H. Qu. Perceptionbased transparency optimization for direct volume rendering. IEEE Transactions on Visualization and Computer Graphics, 15(6), 2009.

[9] N. Chaytor and M. Schmitter-Edgecombe. The ecological validity of neuropsychological tests: A review of the literature on everyday cognitive skills. Neuropsychology review, 13(4):181-197, 2003.

[10] O. S. Collaboration et al. Estimating the reproducibility of psychological science. Science, 349(6251):aac4716, 2015.

[11] J. Collomosse and P. Hall. Painterly rendering using image salience. In Eurographics UK Conference, 2002. Proceedings. The 20th, pp. 122-128. IEEE, 2002.

[12] C. E. Connor, H. E. Egeth, and S. Yantis. Visual attention: bottom-up versus top-down. Current biology, 14(19):R850-R852, 2004.

[13] N. Cowan. The many faces of working memory and short-term storage. Psychonomic bulletin \& review, 24(4):1158-1170, 2017. 
[14] L. J. Cronbach and P. E. Meehl. Construct validity in psychological tests. Psychological bulletin, 52(4):281, 1955.

[15] A. R. Dennis and T. A. Carte. Using geographical information systems for decision making: Extending cognitive fit theory to map-based presentations. Information Systems Research, 9(2):194-203, 1998.

[16] T. Drew, M. L.-H. Võ, and J. M. Wolfe. The invisible gorilla strikes again: Sustained inattentional blindness in expert observers. Psychological science, 24(9): 1848-1853, 2013.

[17] J. S. B. Evans and K. E. Stanovich. Dual-process theories of higher cognition: Advancing the debate. Perspectives on psychological science, 8(3):223-241, 2013.

[18] S. I. Fabrikant, S. R. Hespanha, and M. Hegarty. Cognitively inspired and perceptually salient graphic displays for efficient spatial inference making. Annals of the Association of American Geographers, 100(1):13-29, 2010.

[19] A. Fagerlin, C. Wang, and P. A. Ubel. Reducing the influence of anecdotal reasoning on people's health care decisions: is a picture worth a thousand statistics? Medical decision making, 25(4):398-405, 2005

[20] M. Galesic and R. Garcia-Retamero. Graph literacy: A cross-cultural comparison. Medical Decision Making, 31(3):444-457, 2011.

[21] S. Garden, C. Cornoldi, and R. H. Logie. Visuo-spatial working memory in navigation. Applied Cognitive Psychology: The Official Journal of the Society for Applied Research in Memory and Cognition, 16(1):35$50,2002$.

[22] M. Gattis and K. J. Holyoak. Mapping conceptual to spatial relations in visual reasoning. Journal of Experimental Psychology: Learning, Memory, and Cognition, 22(1):231, 1996.

[23] G. Gigerenzer, P. M. Todd, and A. R. Group. Simple heuristics that make us smart. Oxford University Press, 1999.

[24] E. B. Goldstein and J. Brockmole. Sensation and perception. Cengage Learning, 2016.

[25] S. Haroz and D. Whitney. How capacity limits of attention influence information visualization effectiveness. IEEE Transactions on Visualization and Computer Graphics, 18(12):2402-2410, 2012

[26] M. Hegarty. The cognitive science of visual-spatial displays: Implications for design. Topics in cognitive science, 3(3):446-474, 2011.

[27] M. Hegarty, M. S. Canham, and S. I. Fabrikant. Thinking about the weather: How display salience and knowledge affect performance in a graphic inference task. Journal of Experimental Psychology: Learning, Memory, and Cognition, 36(1):37, 2010.

[28] L. Itti and C. Koch. Target detection using saliency-based attention. Technical report, California Inst of Tech Pasadena Computational and Neural Systems Program, 2000.

[29] H. Jänicke and M. Chen. A salience-based quality metric for visualization. In Computer Graphics Forum, vol. 29, pp. 1183-1192. Wiley Online Library, 2010.

[30] S. Joslyn and J. LeClerc. Decisions with uncertainty: The glass half full. Current Directions in Psychological Science, 22(4):308-315, 2013.

[31] D. Kahneman and P. Egan. Thinking, fast and slow, vol. 1. Farrar, Straus and Giroux New York, 2011.

[32] M. J. Kane, M. K. Bleckley, A. R. Conway, and R. W. Engle. A controlled-attention view of working-memory capacity. Journal of Experimental Psychology: General, 130(2):169, 2001.

[33] C. Kinkeldey, A. M. MacEachren, M. Riveiro, and J. Schiewe. Evaluating the effect of visually represented geodata uncertainty on decisionmaking: systematic review, lessons learned, and recommendations. Cartography and Geographic Information Science, 44(1):1-21, 2017.

[34] S. Kriz and M. Hegarty. Top-down and bottom-up influences on learning from animations. International Journal of Human-Computer Studies, 65(11):911-930, 2007.

[35] M. Lallanilla, April 24, 2014 10:15 am 2014.

[36] L. Liu, A. P. Boone, I. T. Ruginski, L. Padilla, M. Hegarty, S. H. CreemRegehr, W. B. Thompson, C. Yuksel, and D. H. House. Uncertainty visualization by representative sampling from prediction ensembles. IEEE transactions on visualization and computer graphics, 23(9):21652178, 2017.

[37] L. Liu, L. Padilla, S. H. Creem-Regehr, and D. H. House. Visualizing uncertain tropical cyclone predictions using representative samples from ensembles of forecast tracks. IEEE transactions on visualization and computer graphics, 2018.

[38] R. B. Lotto, S. M. Williams, and D. Purves. An empirical basis for mach bands. Proceedings of the National Academy of Sciences, 96(9):5239_ 5244, 1999.

[39] T. Munzner. Visualization analysis and design. CRC press, 2014.

[40] U. Neisser and R. Becklen. Selective looking: Attending to visually specified events. Cognitive psychology, 7(4):480-494, 1975.

[41] L. Padilla, S. H. Creem-Regehr, M. Hegarty, and J. K. Stefanucci. Decision making with visualizations: A cognitive framework across disciplines. Cognitive Research: Principles and Implications, In Press.

[42] L. Padilla, P. S. Quinan, M. Meyer, and S. H. Creem-Regehr. Evaluating the impact of binning $2 \mathrm{~d}$ scalar fields. IEEE transactions on visualization and computer graphics, 23(1):431-440, 2017.

[43] L. M. Padilla. Visual-spatial biases in ensemble cognition. ProQuest, p. 49, 2018.

[44] L. M. Padilla, G. Hansen, I. T. Ruginski, H. S. Kramer, W. B. Thompson, and S. H. Creem-Regehr. The influence of different graphical displays on nonexpert decision making under uncertainty. Journal of Experimental Psychology: Applied, 21(1):37, 2015.

[45] L. M. Padilla, I. T. Ruginski, and S. H. Creem-Regehr. Effects of ensemble and summary displays on interpretations of geospatial uncertainty data. Cognitive research: principles and implications, 2(1):40, 2017.

[46] H. Pashler and C. R. Harris. Is the replicability crisis overblown? three arguments examined. Perspectives on Psychological Science, 7(6):531-536, 2012.

[47] R. E. Patterson, L. M. Blaha, G. G. Grinstein, K. K. Liggett, D. E. Kaveney, K. C. Sheldon, P. R. Havig, and J. A. Moore. A human cognition framework for information visualization. Computers \& Graphics, 42:42-58, 2014.

[48] S. E. Petersen and M. I. Posner. The attention system of the human brain: 20 years after. Annual review of neuroscience, 35:73-89, 2012

[49] S. Pinker. A theory of graph comprehension. Artificial intelligence and the future of testing, pp. 73-126, 1990.

[50] F. Ratliff. Mach bands: quantitative studies on neural networks. Holden-Day, San Francisco London Amsterdam, 1965.

[51] P. M. Rothwell. External validity of randomised controlled trials:to whom do the results of this trial apply?. The Lancet, 365(9453):82-93, 2005.

[52] I. T. Ruginski, A. P. Boone, L. M. Padilla, L. Liu, N. Heydari, H. S. Kramer, M. Hegarty, W. B. Thompson, D. H. House, and S. H. CreemRegehr. Non-expert interpretations of hurricane forecast uncertainty visualizations. Spatial Cognition \& Computation, 16(2):154-172, 2016.

[53] U. Rutishauser, D. Walther, C. Koch, and P. Perona. Is bottom-up attention useful for object recognition? In Computer Vision and Pattern Recognition, 2004. CVPR 2004. Proceedings of the 2004 IEEE Computer Society Conference on, vol. 2, pp. II-II. IEEE, 2004.

[54] J. A. Schirillo and E. R. Stone. The greater ability of graphical versus numerical displays to increase risk avoidance involves a common mechanism. Risk Analysis, 25(3):555-566, 2005.

[55] P. Shah, E. G. Freedman, and I. Vekiri. The comprehension of quantitative information in graphical displays. Cambridge University Press, 2005.

[56] Z. Shipstead, T. L. Harrison, and R. W. Engle. Working memory capacity and the scope and control of attention. Attention, Perception, \& Psychophysics, 77(6):1863-1880, 2015.

[57] D. J. Simons. The value of direct replication. Perspectives on Psychological Science, 9(1):76-80, 2014.

[58] D. J. Simons and C. F. Chabris. Gorillas in our midst: Sustained inattentional blindness for dynamic events. Perception, 28(9):10591074, 1999.

[59] S. A. Sloman. Two systems of reasoning. 2002.

[60] E. R. Stone, W. R. Sieck, B. E. Bull, J. F. Yates, S. C. Parks, and C. J. Rush. Foreground: background salience: Explaining the effects of graphical displays on risk avoidance. Organizational behavior and human decision processes, 90(1):19-36, 2003.

[61] W. Stroebe and F. Strack. The alleged crisis and the illusion of exact replication. Perspectives on Psychological Science, 9(1):59-71, 2014. 
[62] W. Thompson, R. Fleming, S. Creem-Regehr, and J. K. Stefanucci. Visual perception from a computer graphics perspective. AK Peters/CRC Press, 2016.

[63] A. Tversky and D. Kahneman. Judgment under uncertainty: Heuristics and biases. science, 185(4157):1124-1131, 1974.

[64] N. S. Umanath and I. Vessey. Multiattribute data presentation and human judgment: A cognitive fit perspective. Decision Sciences, 25(56):795-824, 1994.

[65] S. K. Ungerleider and L. G. Mechanisms of visual attention in the human cortex. Annual review of neuroscience, 23(1):315-341, 2000.

[66] I. Vessey. Cognitive fit: A theory-based analysis of the graphs versus tables literature. Decision Sciences, 22(2):219-240, 1991

[67] I. Vessey. The effect of information presentation on decision making: A cost-benefit analysis. Information \& Management, 27(2):103-119, 1994.

[68] I. Vessey. The theory of cognitive fit. Human-computer interaction and management information systems: Foundations, pp. 141-183, 2006.

[69] I. Vessey and D. Galletta. Cognitive fit: An empirical study of information acquisition. Information systems research, 2(1):63-84, 1991.

[70] L. Wang, J. Giesen, K. T. McDonnell, P. Zolliker, and K. Mueller. Color design for illustrative visualization. IEEE Transactions on Visualization and Computer Graphics, 14(6):1739-1754, 2008.

[71] E. A. Waters, N. D. Weinstein, G. A. Colditz, and K. Emmons. Formats for improving risk communication in medical tradeoff decisions. Journal of health communication, 11(2):167-182, 2006.

[72] E. A. Waters, N. D. Weinstein, G. A. Colditz, and K. M. Emmons Reducing aversion to side effects in preventive medical treatment decisions. Journal of Experimental Psychology: Applied, 13(1):11, 2007.

[73] B. Zhu and S. A. Watts. Visualization of network concepts: The impact of working memory capacity differences. Information Systems Research, 21(2):327-344, 2010 